\title{
Germ Cell Tumours of Ovary-Surgery: How Much ?
}

\section{SUNESH KUMAR}

Germ cell tumours (GCT) of ovary comprise 2$5 \%$ of all ovarian malignancies. Majority of ovarian GCT occur in young adolescent girls. Advent of successful chemotherapy has changed outlook in the management of Ovarian GCT.Return of menstrual and successful pregnancy outcome is almost a rule in most cases successfully managed.This brings in focus a Conservative Surgical approach while managing cases of Ovarian GCT. Surgery is almost always first step in the management of germ cell tumours of ovary.At the time of laparotomy after care full assessment of extent of disease surgical procedure should comprise of removal of affected ovary,ipsilateral pelvic and para-aortic lymph node sampling,omental
biopsy.Careful palpation of contralateral ovary for any obiovious tumour suffices,avoid bisection of ovary to exclude tumour as it may hamper subsequent fertility by forming periovarian adhesion. Even in cases with bilateral ovarian tumour involvement it may be possible to conserve a part of normal looking ovarian tissue at the time of surgery.Advent of IVF-ETwith oocyte donation has provided hope for child bearing in case both ovaries are to be sacrificed at the time of surgery .Cases are of late being manged by neoadjuvant chemotherapy followed by surgery,such an approach provides better approach for preservation of healty ovarian tissue.

$\overline{\text { Department of Obstetrics \& Gynecology All India Institute of Medical }}$

Sciences, New Delhi-110029

E-mail: kumar.sunesh@yahoo.com 